\title{
Might carbon-atmosphere white dwarfs harbour a new type of pulsating star?
}

\author{
G. Fontaine ${ }^{1}$, P. Brassard ${ }^{1}$, and P. Dufour ${ }^{2}$ \\ 1 Département de Physique, Université de Montréal, CP 6128, Succursale Centre-Ville, Montréal, QC H3C 3J7, Canada \\ e-mail: [fontaine; brassard] aastro.umontreal.ca \\ 2 Department of Astronomy and Steward Observatory, University of Arizona, 933 North Cherry Avenue, Tucson, AZ 85721, USA \\ e-mail: dufourpa@as.arizona.edu
}

Received 26 February 2008 / Accepted 14 March 2008

\begin{abstract}
Aims. In the light of the recent and unexpected discovery of a new type of white dwarfs, those with carbon-dominated atmospheres, we examine the asteroseismological potential of such stars. The motivation behind this is based on the observation that past models of carbon-atmosphere white dwarfs have partially ionized outer layers that bear a strong resemblance to those responsible for mode excitation in models of pulsating DB (helium-atmosphere) and pulsating DA (hydrogen-atmosphere) white dwarfs.

Methods. We carry out a stability survey of models of carbon-atmosphere white dwarfs following a full nonadiabatic approach. To connect with previously known pulsating white dwarfs, we specifically search for excited $g$-modes with $\ell=1$ in the period window 80-1200 s. We concentrate on models with $T_{\text {eff }} \leq 30000 \mathrm{~K}$, a limit below which the real carbon-atmosphere stars are found, and investigate the effects of changing the surface gravity, the composition of the envelope, and the treatment of the convective efficiency. Results. Our main result is that carbon-atmosphere white dwarfs may indeed drive low-order $g$-modes in certain regions of parameter space. For instance, $\log g=8.0$ models characterized by an envelope composition $X(\mathrm{C})=X(\mathrm{He})=0.5$ and ML2 convection, show a broad instability strip in the range $18400-12600 \mathrm{~K}$. This is shifted to $20800-17200 \mathrm{~K}$ for $\log g=9.0$ models. In this interval of surface gravity, the excited periods are found between $\sim 100 \mathrm{~s}$ and $\sim 700 \mathrm{~s}$, and the shorter periods are excited in the higher-gravity models. Adding carbon in the envelope mixture tends to extinguish pulsational driving.

Conclusions. Given the right location in parameter space, some carbon-atmosphere white dwarfs are predicted to show pulsational instability against gravity modes. We are eagerly awaiting the results of observational searches for luminosity variations in these stars.
\end{abstract}

Key words. stars: oscillations - stars: white dwarfs

\section{Introduction}

Dufour et al. (2007) reported on the unexpected discovery of a new type of white dwarf. These are stars with atmospheres dominated by carbon. Until then, white dwarfs cooler than $\sim 80000 \mathrm{~K}$ were known to come in only two flavors, those with almost pure hydrogen atmospheres (the so-called DA stars comprising some 75 to $80 \%$ of the known sample), and those with almost pure helium atmospheres (the non-DAs accounting for the rest). Obviously quite rare since none had ever been found before among the several thousands white dwarfs known, the carbon-atmosphere stars uncovered by Dufour et al. (2007) form a small sample of nine faint objects with $g \sim 18-19$ culled from the Sloan Digital Sky Survey (SDSS). They are all found in a rather narrow range of effective temperature, between $18000 \mathrm{~K}$ and $23000 \mathrm{~K}$. A typical value of the abundance ratio derived from the less noisy SDSS spectra in the sample is $X(\mathrm{C}) / X(\mathrm{He}) \geq$ 99. However, a ratio as small as $X(\mathrm{C}) / X(\mathrm{He}) \geq 3$ cannot be excluded in some of the other spectra according to the more detailed analysis reported by Dufour et al. (2008).

Beyond their very existence, which remains to be explained (see, in this context, the qualitative evolutionary scenario proposed by Dufour et al. 2007), carbon-atmosphere white dwarfs have attracted our interest from another point of view. And indeed, we noticed that past models of carbon-atmosphere white dwarfs in the range of effective temperature where the real ones are found are characterized by an important outer superficial convection zone, very similar to that found in the pulsating DB (V777 Her) stars (centered around $T_{\text {eff }} \simeq 25000 \mathrm{~K}$ ) or in the pulsating DA (ZZ Ceti) stars (found around $T_{\text {eff }} \simeq 12000 \mathrm{~K}$ ). Hence, it follows that carbon-atmosphere white dwarfs could also excite pulsation modes through the same partial ionization/convective driving phenomenon that is at work in these two distinct families of pulsating white dwarfs.

Figure 7 of Fontaine \& Van Horn (1976) compares properties of the convection zones found in models of hydrogen-, helium-, and carbon-atmosphere white dwarfs. There are obvious similarities, and this strongly suggests that a stability study for carbonatmosphere stars would be revealing. In 1974, Fontaine published in his Ph.D. Thesis an examination of white dwarf envelope models, including carbon models, and those are now shown to be of interest in the present context. At the time, only the hydrogen- and helium-atmosphere models were thought to be "relevant". And indeed, to our knowledge, the carbon envelope models of Fontaine \& Van Horn (1976) have remained the only ones of the kind to have been published.

With this background in mind, we decided to investigate the asteroseismological potential of models of carbon-atmosphere white dwarfs. Further impetus came from the involvement of one 
of us (P.D.) with other collaborators to search observationally for luminosity variations in these stars. It is timely to provide the theoretical background in parallel with that search.

\section{Equilibrium models and stability survey}

In the sort of exploratory work presented in this paper, we study relatively simple models of carbon-atmosphere white dwarfs. We thus considered equilibrium structures made of a uniformcomposition envelope sitting on a $\mathrm{C} / \mathrm{O}$ core (also with a uniform composition specified by $X(\mathrm{C})=X(\mathrm{O})=0.5)$. As a working definition of a "carbon-dominated" mixture, we adopted the convention $X(\mathrm{C}) \geq 0.5$ for the mass fraction of carbon in the envelope/atmosphere. Hence, we first constructed stellar models with an envelope defined by $X(\mathrm{C})=X(\mathrm{He})=0.5$, representing a limiting case in the composition domain for carbonatmosphere white dwarfs. In a second step, and to provide a measure of the importance of varying the carbon abundance, we also considered envelope compositions defined by $X(\mathrm{C})=0.75$ and $X(\mathrm{He})=0.25, X(\mathrm{C})=0.9$ and $X(\mathrm{He})=0.1$, as well as $X(\mathrm{C})=0.99$ and $X(\mathrm{He})=0.01$. We explicitly ignored the possible presence of small traces of hydrogen in our survey.

The white dwarf models that we used are static, but full stellar structures nevertheless. To compute them, we took advantage of the scaling law $L(r) \propto M(r)$, as appropriate for a cooling white dwarf (see Brassard \& Fontaine 1994, for more details on this). Given a composition profile, a model is further specified by its effective temperature $T_{\text {eff }}$ and surface gravity $\log g$. Equivalently, due to the specific mass-radius relationship that applies to white dwarfs, the total mass could be specified instead of the surface gravity, but we prefer the first alternative because it connects better with spectroscopy. In addition, in the range of effective temperature of interest here, convection is present in the envelope of carbon-atmosphere white dwarfs (Fontaine \& Van Horn 1976), and we described it through the so-called ML2 version of the mixing-length theory (see Table 1 of Fontaine et al. 1981, for the original definition of ML2). We also briefly explored the effects of changing the modelling of convection by computing some extra models under the assumption of ML3 convection (a version similar to ML2 in terms of the geometry of the eddies, but assuming a ratio of the mixing length to the the local pressure scale height of $\alpha=2$ instead of 1 as in ML2).

We carried out a stability survey of these white dwarf models with our finite-element pulsation codes (Brassard et al. 1992a; Fontaine et al. 1994). Given what is known about V777 Her and ZZ Ceti pulsators, we specifically searched for unstable dipole $(\ell=1)$ modes in the range of periods from $80 \mathrm{~s}$ to $1200 \mathrm{~s}$. These would be $g$-modes. We also concentrated on models cooler than $T_{\text {eff }}=30000 \mathrm{~K}$ to connect with the observed carbon-atmosphere white dwarfs of Dufour et al. (2007). We point out that we used a full nonadiabatic approach, but within the so-called frozen convection approximation. This approximation leads to quite reasonable estimates of the location of the blue edge of the ZZ Ceti instability strip in the $\log g-T_{\text {eff }}$ plane (see, e.g., the discussion in Brassard \& Fontaine 1999), and the same is true for the V777 Her instability strip (Beauchamp et al. 1999). At the same time, it is well known that the frozen convection approximation leads to red edges of instability strips that are cooler, and sometimes much cooler than they ought to be. This caveat should be kept in mind.

A last remark about our equilibrium models concerns our choice for the thickness of the outer carbon-dominated envelope, which we chose at a fractional mass depth of $\log q_{\text {env }} \equiv$ $\log \left(1-M(r)_{\mathrm{env}} / M_{*}\right)=-3.0$ in all cases. At this depth, the

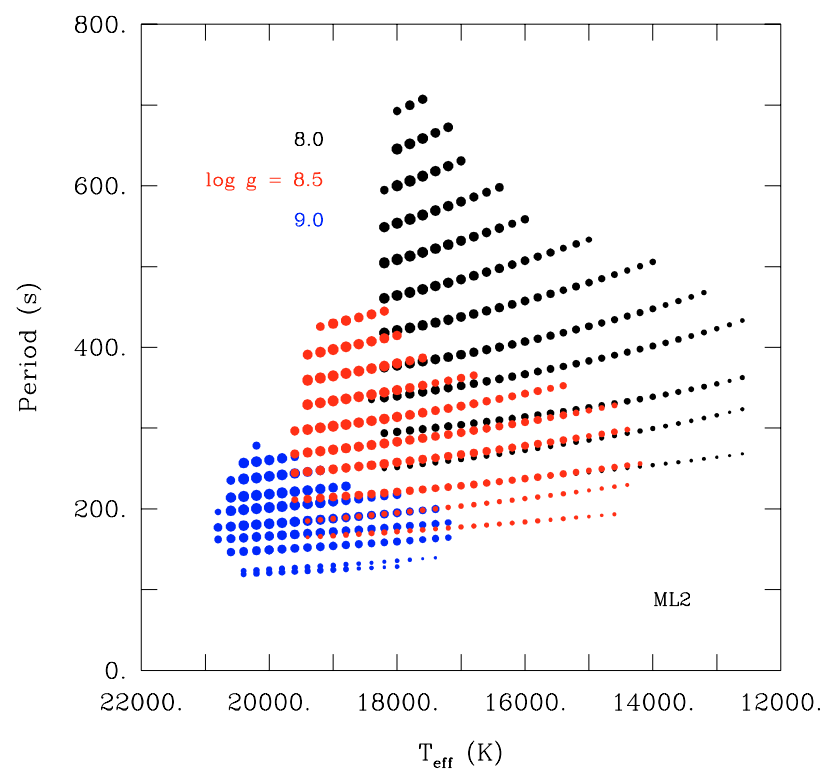

Fig. 1. Period spectra of excited dipole modes in carbon-atmosphere white dwarf models. Three families of models are shown differing in surface gravity (see the color code), but they otherwise have the same envelope composition $(X(\mathrm{C})=X(\mathrm{He})=0.5)$ and ML2 convection. Each dot gives the period of a mode, and its size represents a logarithmic measure of the modulus of the imaginary part $\sigma_{\text {I }}$ of the complex eigenfrequency. The larger the dot, the more unstable the mode.

specified uniform envelope composition changes smoothly to the core composition of $X(\mathrm{C})=X(\mathrm{O})=0.5$ in our models. Clearly, the actual pulsation periods must depend on this particular choice due to trapping effects at the composition transition layer (see, e.g., Brassard et al. 1992b). However, as discussed at some length in Quirion et al. (2004) in the context of GW Vir white dwarfs, it is not so much the actual periods themselves that matter in the kind of stability study that we carried out, but the range of unstable periods. As explicitly demonstrated by Quirion et al. (2004), that interval of unstable periods is very largely independent of the actual choice of $\log q_{\text {env }}$, as long as the base of the envelope is located much deeper than the driving/damping region. The specific choice of $\log q_{\mathrm{env}}=-3.0$ ensures just that.

Figure 1 summarizes some of our results for models with an envelope composition specified by $X(\mathrm{C})=X(\mathrm{He})=0.5$ and ML2 convection. The physical background briefly reported on above is consistent with our finding of pulsation modes that can be excited in some carbon-atmosphere white dwarf models. This finding potentially opens the door to the application of the tools of asteroseismology for the further study of this new class of carbon-atmosphere white dwarfs. The black dots in Fig. 1 map the expected instability strip for a reference family of models with $\log g=8.0$. Dipole modes in this family are excited in a range of effective temperature from $18400 \mathrm{~K}$ to $12600 \mathrm{~K}$. For the reasons given above, the red edge is probably calculated cooler than it ought to be, but the blue edge calculations are reliable. The excited modes are all low-order $g$-modes, with a radial order $k=2$ for the lowest series of adjacent points (separated by $200 \mathrm{~K}$ in $T_{\text {eff }}$ ), to $k=13$ for the top three points illustrated. The total interval of excited periods that is mapped for this family of models covers $\sim 200 \mathrm{~s}$ to $\sim 700 \mathrm{~s}$. The e-folding times of the excited modes illustrated in the figure are all much smaller than the typical evolutionary timescale of a carbon-atmosphere white dwarf, a few times $10^{8} \mathrm{yrs}$, reaching in some cases (the 


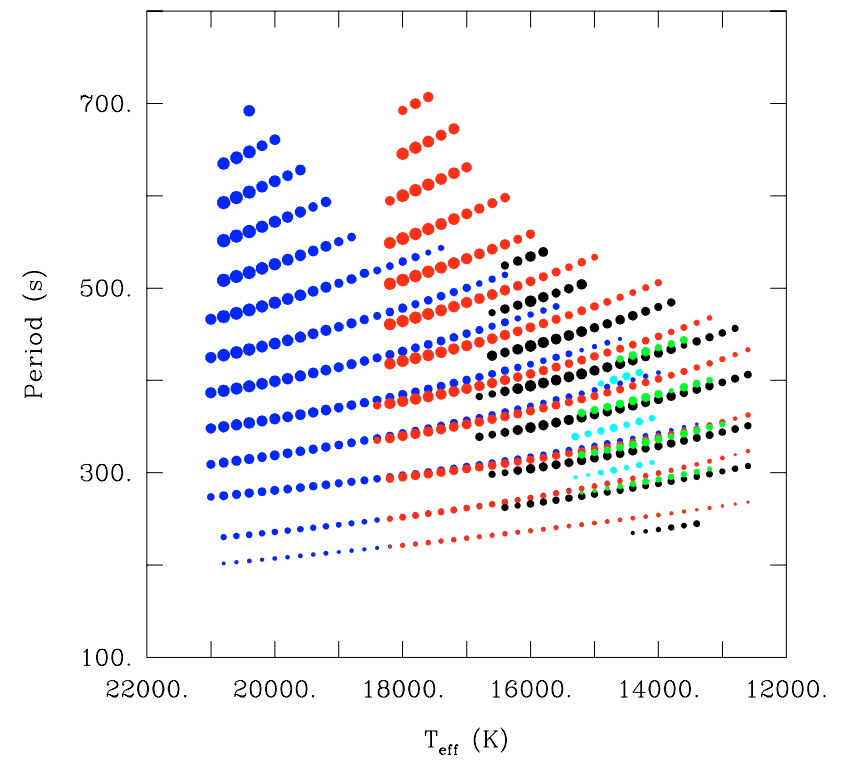

Fig. 2. Similar to Fig. 1, but the red dots refer, this time, to the reference family of models specified by $\log g=8.0, X(\mathrm{C})=X(\mathrm{He})=0.5$, and ML2 convection. The blue dots were obtained from similar models but using the more efficient ML3 version of the mixing-length theory. The black (green, cyan) points again refer to $\log g=8.0$, ML2 models, but with an envelope composition defined by $X(\mathrm{C})=0.75$ and $X(\mathrm{He})=$ $0.25(X(\mathrm{C})=0.9$ and $X(\mathrm{He})=0.1, X(\mathrm{C})=0.99$ and $X(\mathrm{He})=0.01)$.

largest dots in the plot) values as low as 50 to $100 \mathrm{yrs}$. This means that such modes have plenty of time to develop an observable amplitude.

The theoretical blue edge for the $\log g=8.0$ family of models displayed in Fig. 1 may fall short if we examine the current sample of nine carbon-atmosphere white dwarfs discovered by Dufour et al. (2007), since those cluster in a range of $T_{\text {eff }}$ from $23000 \mathrm{~K}$ to $18000 \mathrm{~K}$. However, a simple shift in surface gravity easily pushes the blue edge of the instability strip well within that range, as shown by the red and blue dots in Fig. 1, referring, respectively, to a family of models with $\log g=8.5$ and $\log g=9.0$. Note that this displacement of the blue edge toward higher effective temperatures is accompanied by a shift of the range of unstable periods toward shorter values. This is not surprising because the periods themselves (see, e.g., Brassard et al. 1992 b, in the case of ZZ Ceti pulsators), i.e., those of given $k$ values, are known to decrease when the surface gravity of a white dwarf increases. A fortiori, this is also true for excited periods. The fact that the blue edge temperature increases with increasing surface gravity is also observed and well understood for the pulsating DB (Beauchamp et al. 1999) and the pulsating DA white dwarfs (see, e.g., Gianninas et al. 2006).

Figure 2 shows some other results of our exploratory survey, in particular the effects of changing the chemical composition of the envelope. The red dots correspond to our reference family of models with $\log g=8.0$, ML2 convection, and $X(\mathrm{C})=X(\mathrm{He})=0.5$ in the envelope. All other things being the same, the black dots show a shrinking instability domain when the envelope composition is changed to $X(\mathrm{C})=0.75$ and $X(\mathrm{He})=0.25$. There is a decrease of the blue edge temperature from $18400 \mathrm{~K}$ to $16800 \mathrm{~K}$ in that case. The band of predicted unstable dipole modes also reduces to the range $\sim 230 \mathrm{~s}$ to $\sim 580 \mathrm{~s}$. This trend continues to be verified in models with an envelope composition defined by $X(\mathrm{C})=0.9$ and $X(\mathrm{He})=0.1$ as indicated by the green dots. The predicted blue edge for this family of $\log g=8.0$, ML2 models is now at a value $T_{\text {eff }}=$ $15300 \mathrm{~K}$. Although the location of the blue edge does not change much more, the overall instability domain shrinks further as can be seen from the cyan dots associated with models with a very rich carbon composition in the envelope, i.e., $X(\mathrm{C})=0.99$ and $X(\mathrm{He})=0.01$. This is strong indication that the actual amount of carbon in the envelope of real carbon-atmosphere white dwarfs will be a decisive factor in the fate of these stars as pulsators. Finally, the blue dots in Fig. 2 illustrate the significant blueward shift (some $2600 \mathrm{~K}$ ) of the predicted instability strip and some widening of the instability domain when convection is modelled in terms of the more efficient ML3 version compared to our standard ML2 flavor.

We now examine the question of the mechanism responsible for exciting pulsation modes in these models. Figure 3 illustrates the details of the driving/damping region in two models from the $\log g=8.0$ family above, one $\left(T_{\text {eff }}=17000 \mathrm{~K}\right)$ featuring excited $g$-modes, and the other $\left(T_{\text {eff }}=20000 \mathrm{~K}\right)$ hotter than the blue edge and showing only stable modes. Out of the many $g$-modes found excited in the $17000 \mathrm{~K}$ model (left panel), we have singled out a representative one with indices $k=8$ and $\ell=1$. It has a period of $489.1 \mathrm{~s}$. We also retained the same mode, this time stable and with a period of $451.8 \mathrm{~s}$, in the $20000 \mathrm{~K}$ model (right panel). The figure illustrates a situation comparable to the case of the V777 Her and ZZ Ceti pulsators, but is more complicated because of the presence of two maxima in the opacity distribution instead of a single peak in these other pulsators. In the present case, both opacity maxima (caused by two distinct partial ionization zones in the envelope mixture) are "active" in the sense that they both contribute to the driving/damping process. What we can observe from the plot is that the regions on the descending side (going in from the surface) of an opacity bump contribute locally to driving, while the deeper adjacent zones, where the opacity plummets to relatively low values, contribute instead to damping. In the $17000 \mathrm{~K}$ model, the two opacity bumps are relatively close to each other and are part of a single convection zone. The damping region between the two bumps is then relatively narrow (see the left panel) and the overall work integral comes out positive, meaning that the mode is globally excited. In contrast, the two opacity maxima are relatively far apart in the $20000 \mathrm{~K}$ model, so they lead to the formation of two distinct, separate convection zones. In that case, there is a much larger region between the opacity bumps where radiative damping becomes so large that the overall work integral comes out negative.

\section{Conclusion}

Our exploratory survey of the asteroseismological potential of carbon-atmosphere white dwarfs has allowed us to uncover regions of parameter space where that potential can be fulfilled. For example, low-order $g$-modes, typical of other pulsating white dwarfs such as the V777 Her and ZZ Ceti stars, can be excited in a wide range of effective temperature $(18400 \mathrm{~K}$ to $12600 \mathrm{~K}$ ) for models specified by $\log g=8.0$, ML2 convection, and an envelope composition given by $X(\mathrm{C})=X(\mathrm{He})=$ 0.5 . Hotter models also can become unstable if their surface gravity is increased or if the assumed convective efficiency used in the model building phase is increased. The actual amount of carbon in the envelope is crucial in determining the location and extent of the instability strip. The extent of that domain tends to diminish when the carbon abundance is increased. If real pulsating stars are found in the current sample of carbon-atmosphere white dwarfs, we would expect them to be massive, to show 

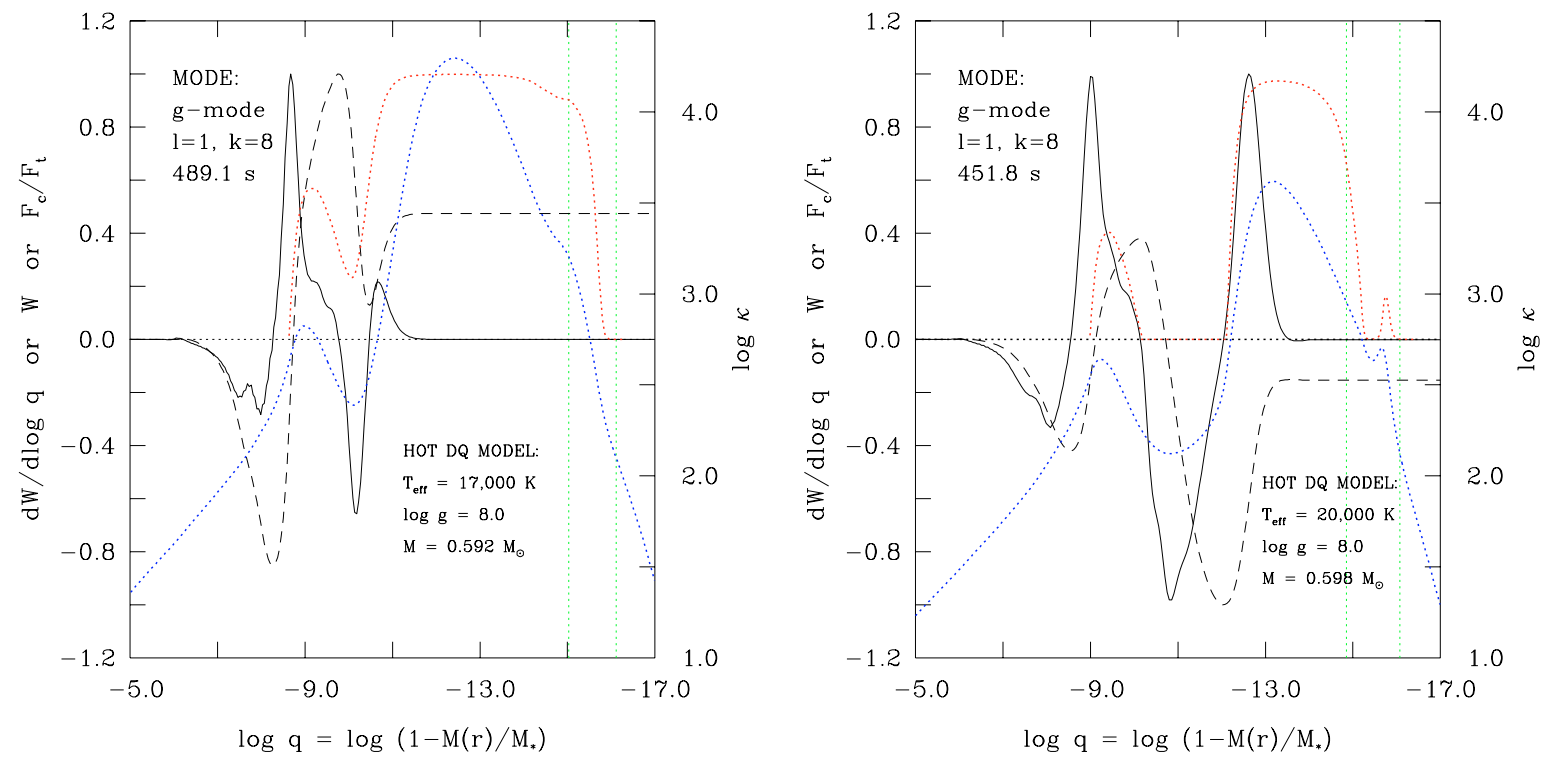

Fig. 3. Left panel: details of the driving/damping process for a typical $g$-mode excited in a $17000 \mathrm{~K}$, ML2 model of a hot DQ (carbon-atmosphere) white dwarf with a envelope composition $X(\mathrm{C})=X(\mathrm{He})=0.5$ and a gravity $\log g=8.0$. The solid curve shows the integrand of the work integral of the mode as a function of fractional mass depth. The dashed curve shows the running work integral, from left to right, toward the surface of the model. The red dotted curve shows the ratio of the convective to total flux. The blue dotted curve gives the run of the Rosseland opacity, to be read on the RHS ordinate axis. The radiative opacity values were taken from the OPAL data base. The maximum in the opacity profile, located at $\log q \simeq-12.41$ and corresponding to a temperature $T \simeq 1.045 \times 10^{5} \mathrm{~K}$, is caused by the partial ionization of He II, C III, and C IV in the envelope mixture. The secondary maximum, located at $\log q \simeq-8.98$ and corresponding to a temperature $T \simeq 1.177 \times 10^{6} \mathrm{~K}$, is caused by the partial ionization of C V, and C VI. The vertical green dotted line on the left (right) gives the location of the base of the atmosphere at optical depth $\tau_{\mathrm{R}}=100$ (of the phoptosphere at $\tau_{\mathrm{R}}=2 / 3$ ). Right panel: similar and for the same $g$-mode, but in a hotter model at $20000 \mathrm{~K}$ for which all modes investigated are stable. It can be seen that the value of the work integral $W$ at the surface (dashed curve) is now negative, contrary to the case in the left panel, and this signifies that the mode is not excited. Note also the two separate convection zones in this model.

significant amounts of helium in their atmospheres, and to possibly be undergoing efficient convective transport.

Here we have provided the basic theoretical framework within which to interpret possible discoveries of pulsations in carbon-atmosphere white dwarfs. At least one observational search is currently pursuing that. The sample of carbonatmosphere stars of Dufour et al. (2007) is small, but it is likely, now that we know how to recognize them, that more will be uncovered from future data releases from the SDSS. If real pulsators are found among that group, they will constitute the fourth distinct category of pulsating white dwarfs after the GW Vir, V777 Her, and ZZ Ceti stars. More detailed pulsation studies will certainly be warranted if that is the case.

Acknowledgements. This work was supported in part by the NSERC of Canada. G.F. also gratefully acknowledges the contribution of the Canada Research Chair Program.

\section{References}

Beauchamp, A., Wesemael, F., Bergeron, P., et al. 1999, ApJ, 516, 887 Brassard, P., \& Fontaine, G. 1994, in The Equation of State in Astrophysics, ed. G. Chabrier, \& E. Schatzman (Cambridge: Cambridge University Press), IAU Colloq., 147, 560

Brassard, P., \& Fontaine, G. 1999, in Theory and Tests of Convection in Stellar Structure, ed. A. Giminez, E. F. Guinan, \& B. Montesinos (San Francisco: ASP), ASP Conf. Ser., 173, 329

Brassard, P., Fontaine, G., Wesemael, F., \& Tassoul, M. 1992b, ApJS, 81, 747

Brassard, P., Pelletier, C., Fontaine, G., \& Wesemael, F. 1992a, ApJS, 80, 725

Dufour, P., Liebert, J., Fontaine, G., \& Behara, N. 2007, Nature, 450, 522

Dufour, P., et al. 2008, in preparation

Fontaine, G. 1974, Ph.D. Thesis, University of Rochester

Fontaine, G., \& Van Horn, H. M. 1976, ApJS, 31, 467

Fontaine, G., Villeneuve, B., \& Wilson, J. 1981, ApJ, 243, 550

Fontaine, G., Brassard, P., Wesemael, F., \& Tassoul, M. 1994, ApJ, 428, L61

Gianninas, A., Bergeron, P., \& Fontaine, G. 2006, AJ, 132, 831

Quirion, P.-O., Fontaine, G., \& Brassard, P. 2004, ApJ, 610, 436 